\title{
Engineering Supramolecular Nanomedicine for Targeted Near Infrared-Triggered Mitochondrial Dysfunction to Potentiate Cisplatin for Efficient Chemo-Phototherapy
}

Wei Zhang ${ }^{1, \#}$, Xiang-Fu Du ${ }^{1,2, \#, ~ B e n ~ L i u ~}{ }^{1}$, Cairong Li ${ }^{1}$, Jing Long ${ }^{1}$, Mei-Xia Zhao ${ }^{2, *}$, Zhenyu Yao ${ }^{1}$, Xing-Jie Liang ${ }^{3,},{ }^{*}$ Y uxiao Lai ${ }^{1,{ }^{*}}$

${ }^{1}$ Centre for Translational Medicine Research \& Development, Shenzhen Institute of Advanced Technology, Chinese Academy of Sciences, Shenzhen 518055, China

${ }^{2}$ Key Laboratory of Natural Medicine and Immune Engineering, Henan University, Jinming Road, Kaifeng 475004, China

${ }^{3}$ CAS Center for Excellence in Nanoscience, CAS Key Laboratory for Biomedical Effects of Nanomaterials and Nanosafety, National Center for Nanoscience and Technology, Beijing 100190, China

Corresponding Author:

Prof. Mei-Xia Zhao, Email: zhaomeixia2011@henu.edu.cn

Prof. Xing-Jie Liang, Email: liangxj@nanoctr.cn

Prof. Yuxiao Lai, Email: yx.lai@siat.ac.cn

\# These authors contributed equally to this work. 


\section{Supplementary Figures}

(A)
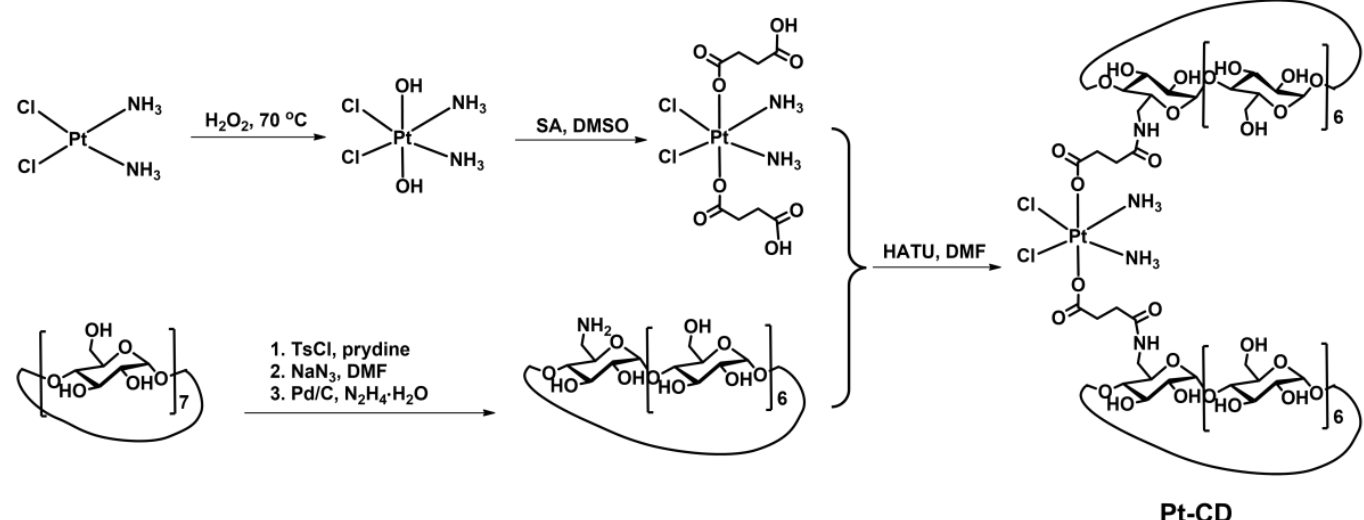

HATU, DMF
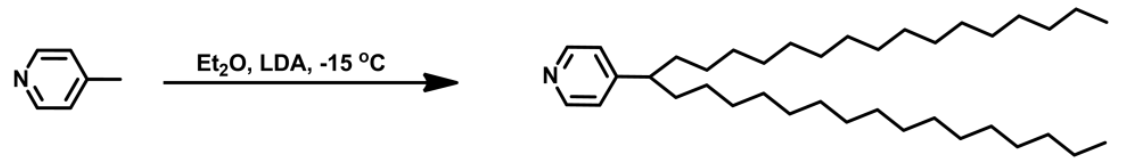

(B)

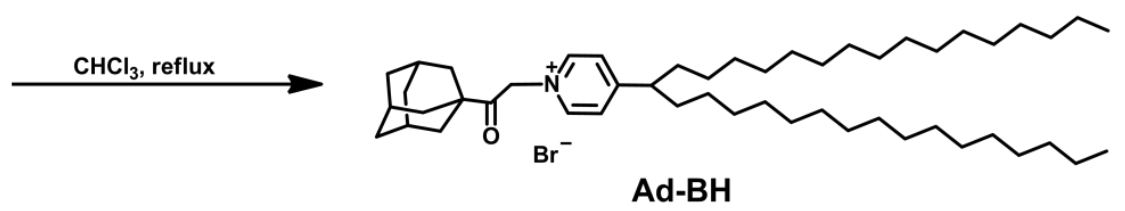

Scheme 1. The synthesis routes for (A) Pt-CD and (B) Ad-BH.

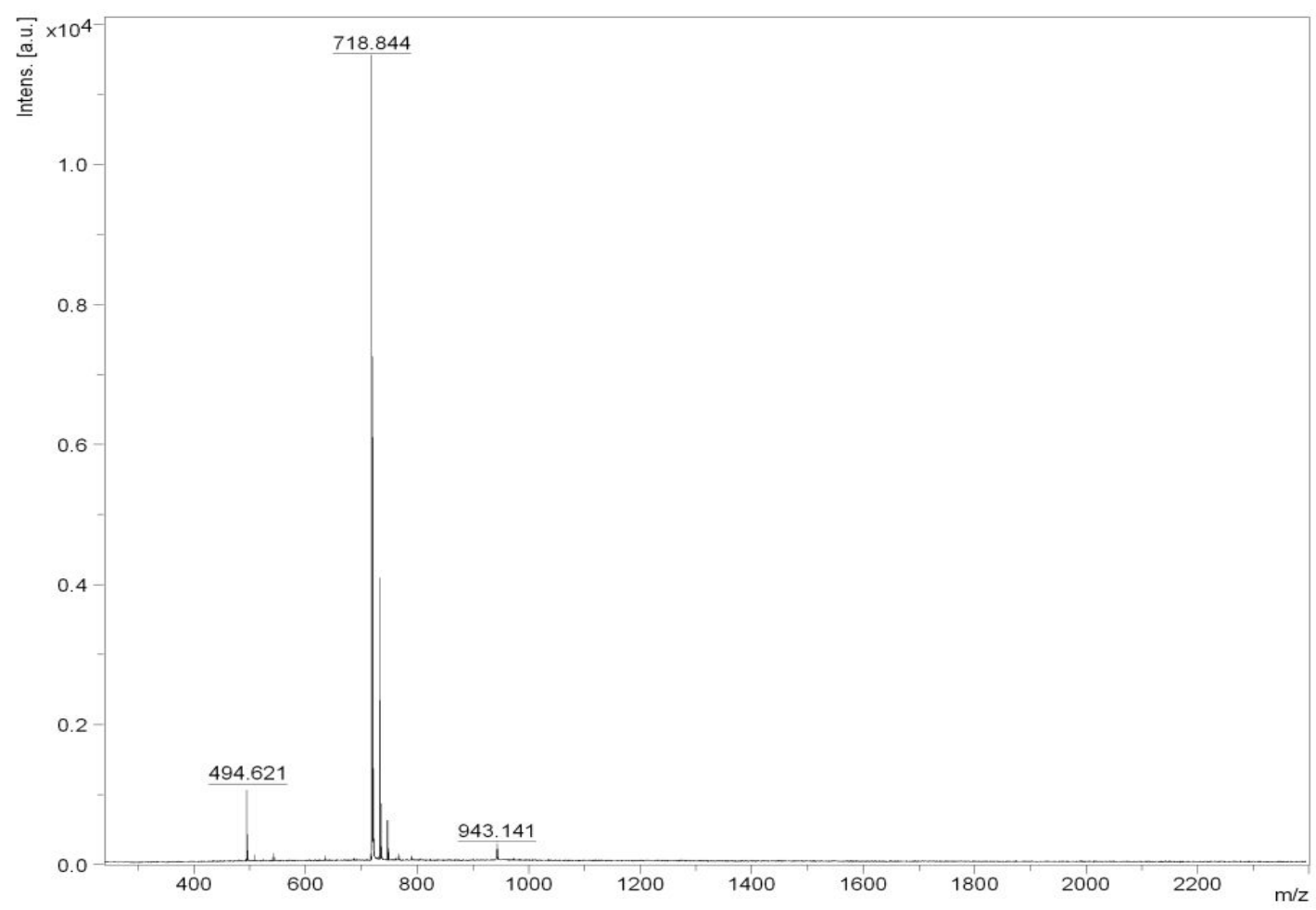

Figure S1. Mass spectrum of Ad-BH. 


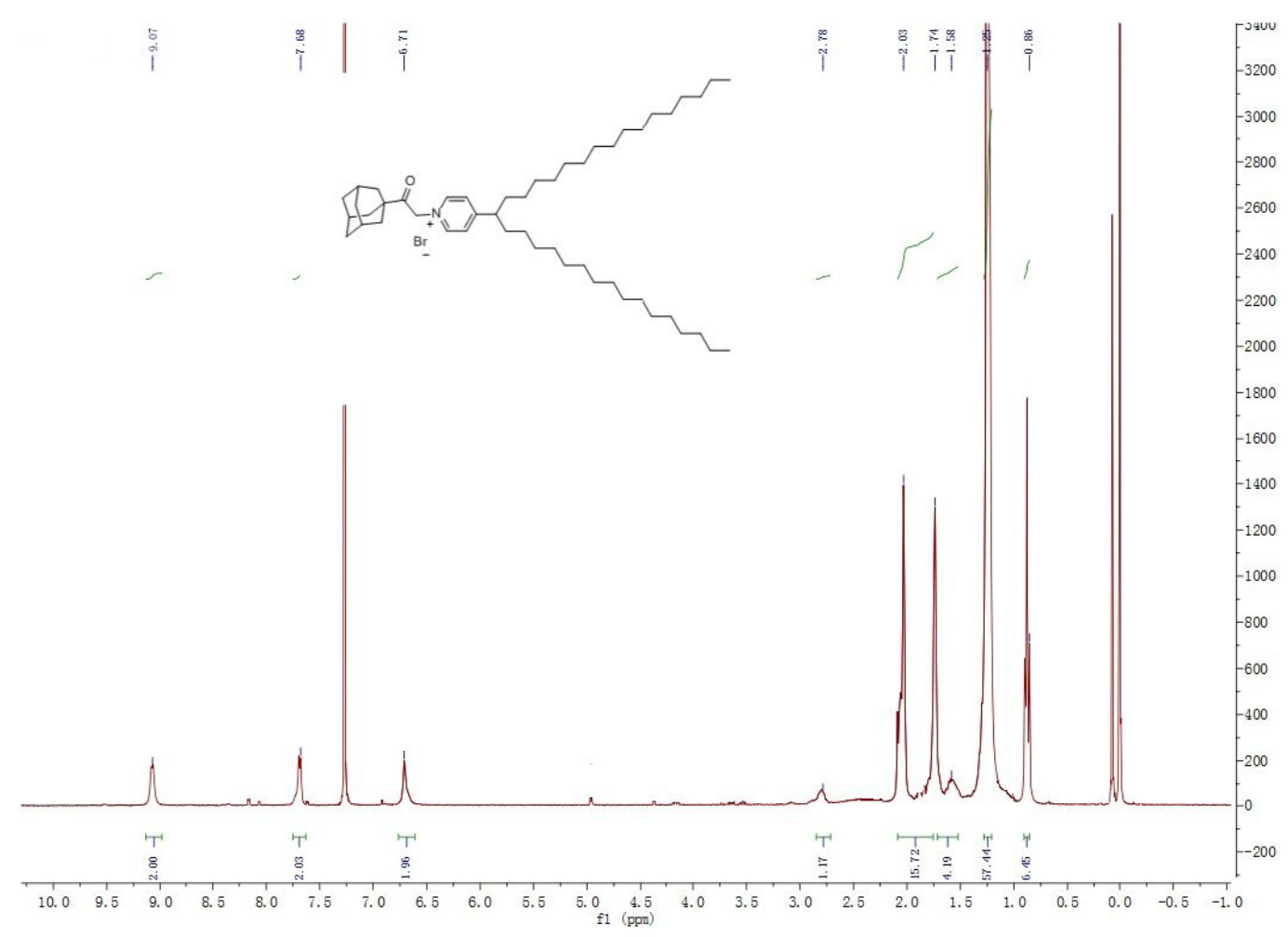

Figure S2. The 1H NMR spectrum of Ad-BH.

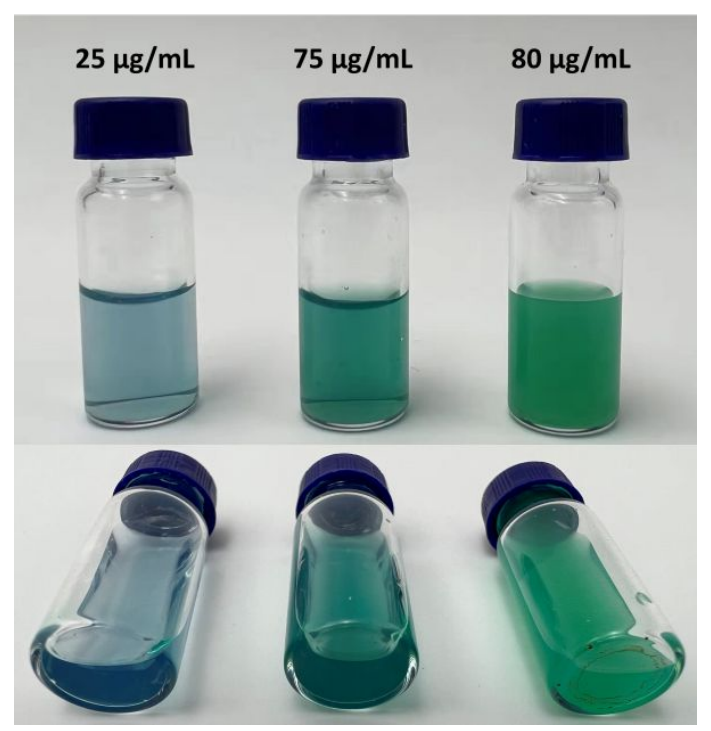

Figure S3. The digital images of the solutions of Pt-CD, Ad-BH and IR780 with different concentrations for preparation of IR780@Pt NPs. In each vial, 4 ml of Pt-CD (12 mg) was added with Ad-Bh (3.1 mg in acetone), and IR780 solution of various amount was added to afford the mixture solution with final IR780 concentration of 25 , 75 or $80 \mu \mathrm{g} / \mathrm{ml}$. 


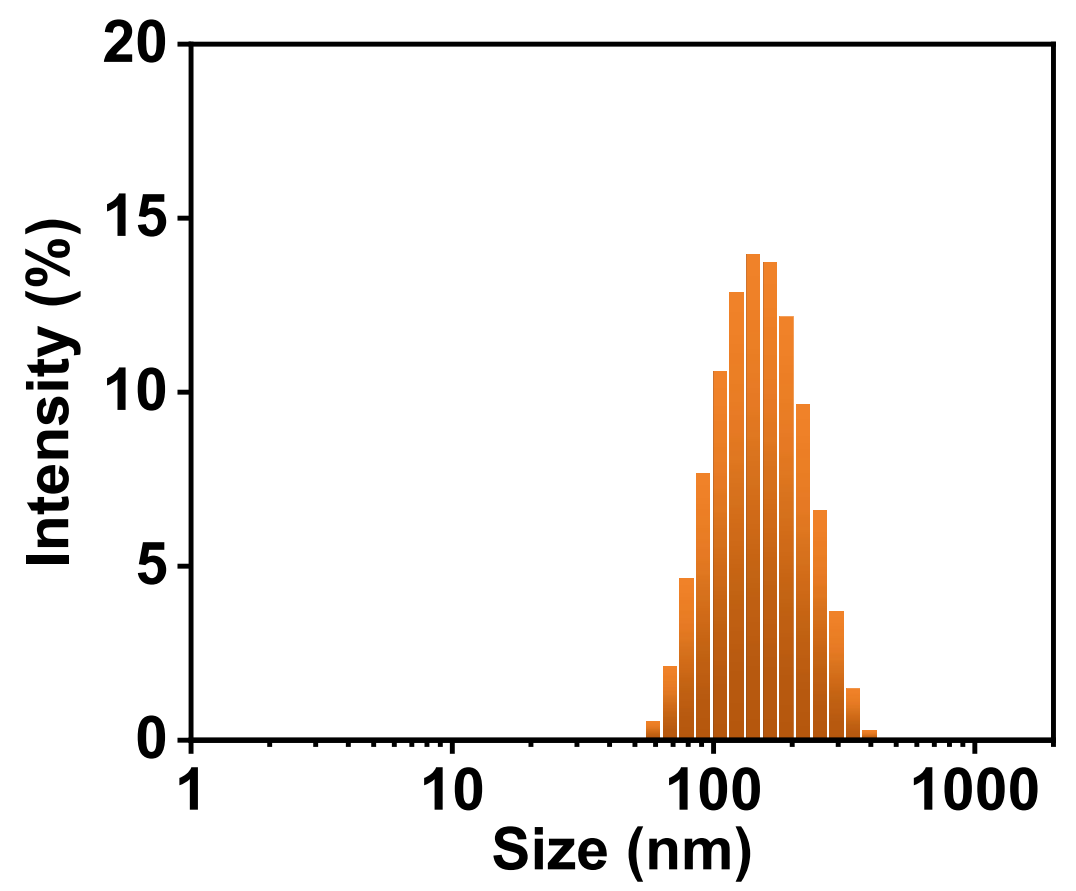

Figure S4. The hydrodynamic size of IR780@Pt NPs measured by Zeta sizer.
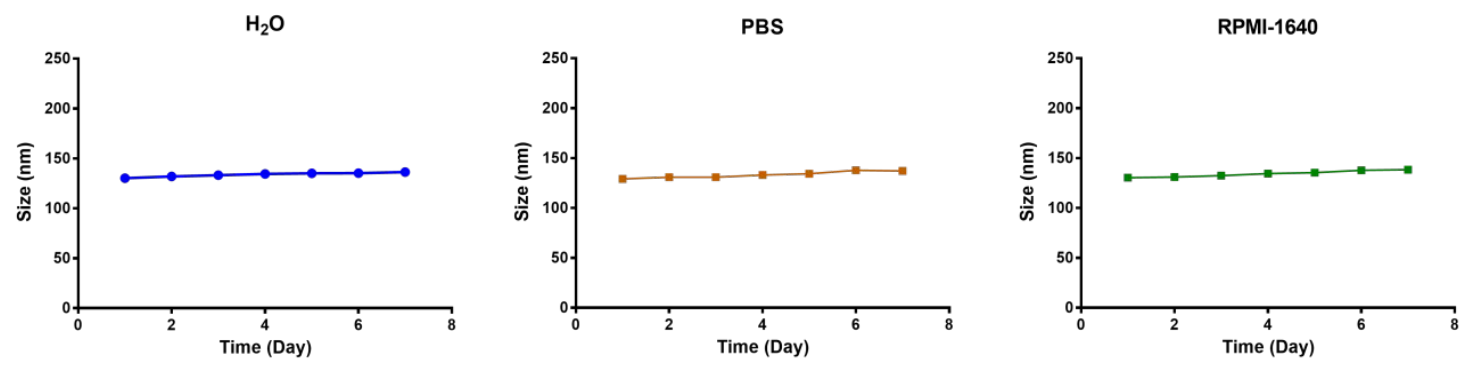

Figure S5. The hydrodynamic diameter of IR780@Pt NPs in different solutions monitored by DLS for 7 days. 


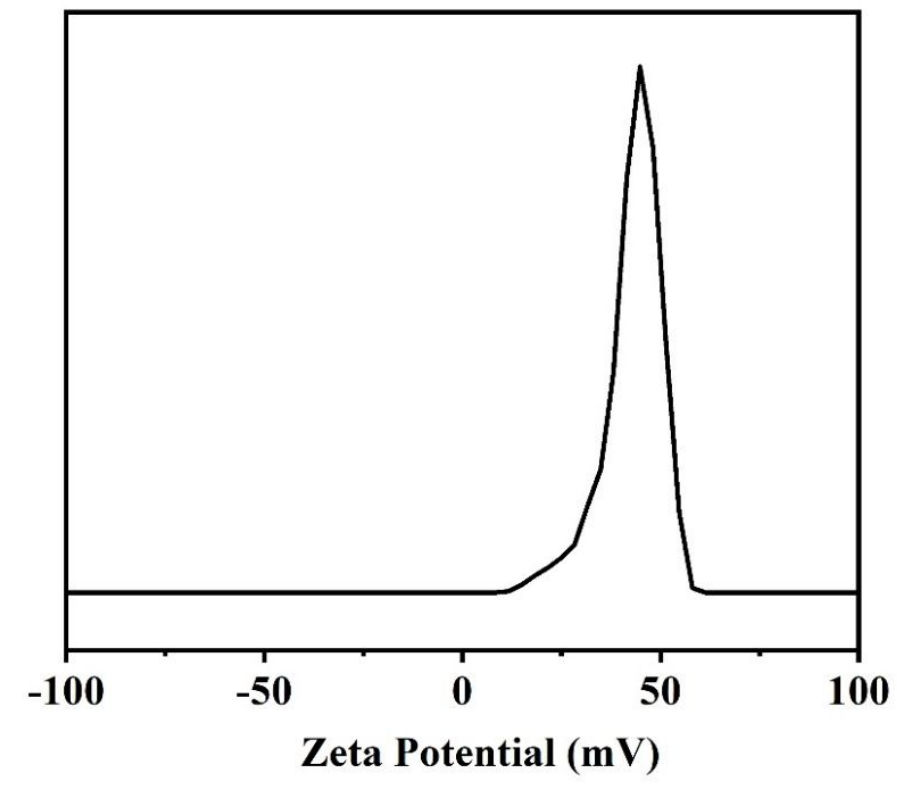

Figure S6. The Zeta potential of IR780@Pt NPs measured by Zeta sizer.

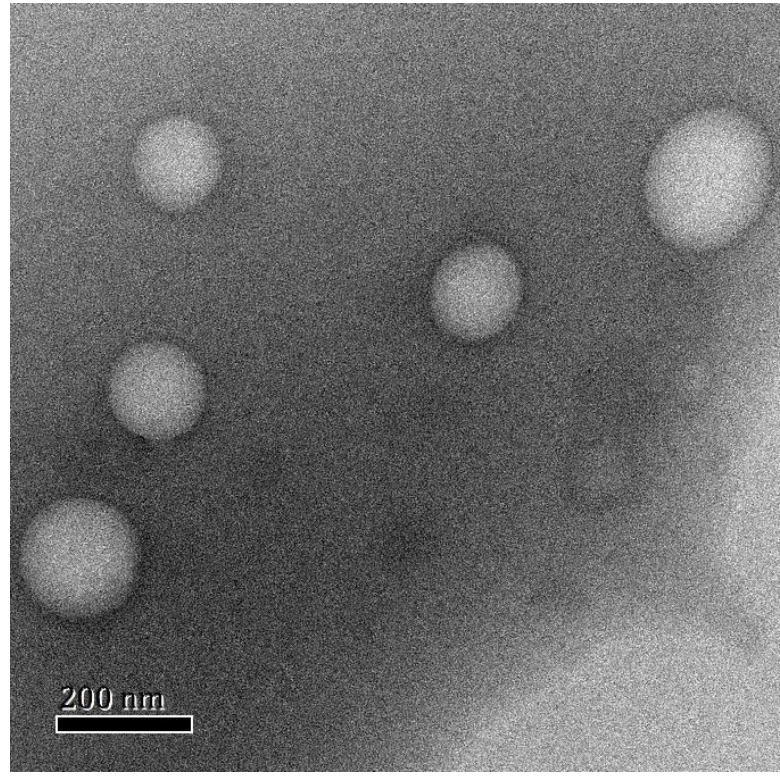

Figure S7. The TEM image of IR780@Pt NPs at higher magnification. 


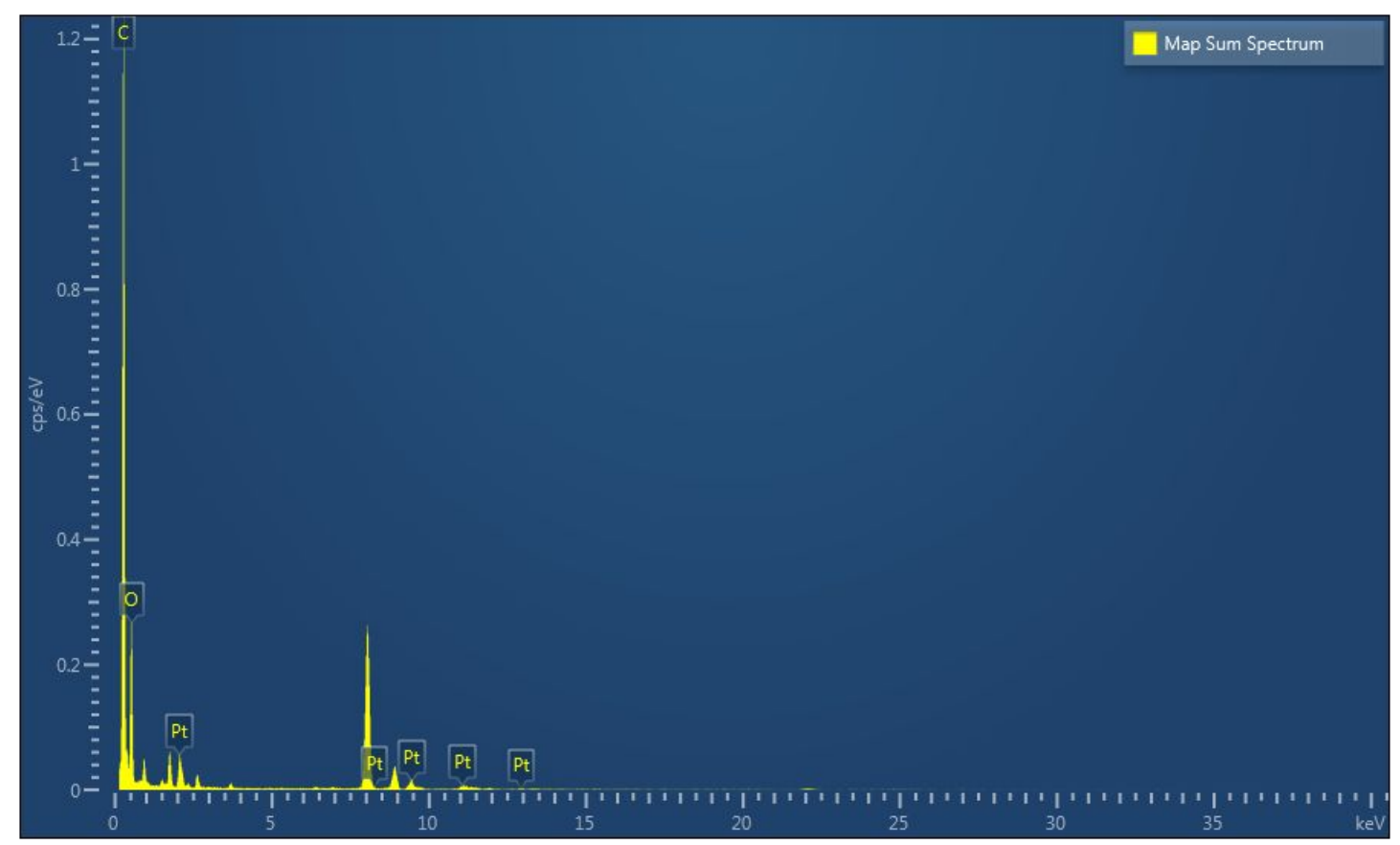

Figure S8. The TEDS spectrum for elemental analysis of IR780@Pt NPs.

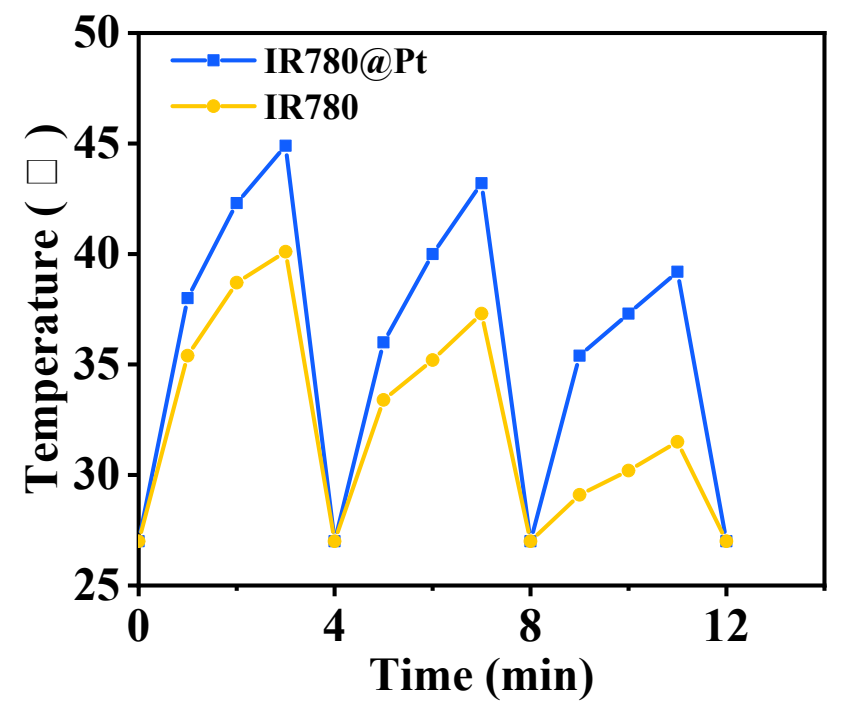

Figure S9. The photothermal stability study for free IR780 and IR780@Pt NPs during three laser on/off cycles. Laser irradiation condition: $808 \mathrm{~nm}, 1 \mathrm{~W} / \mathrm{cm}^{2}$. 

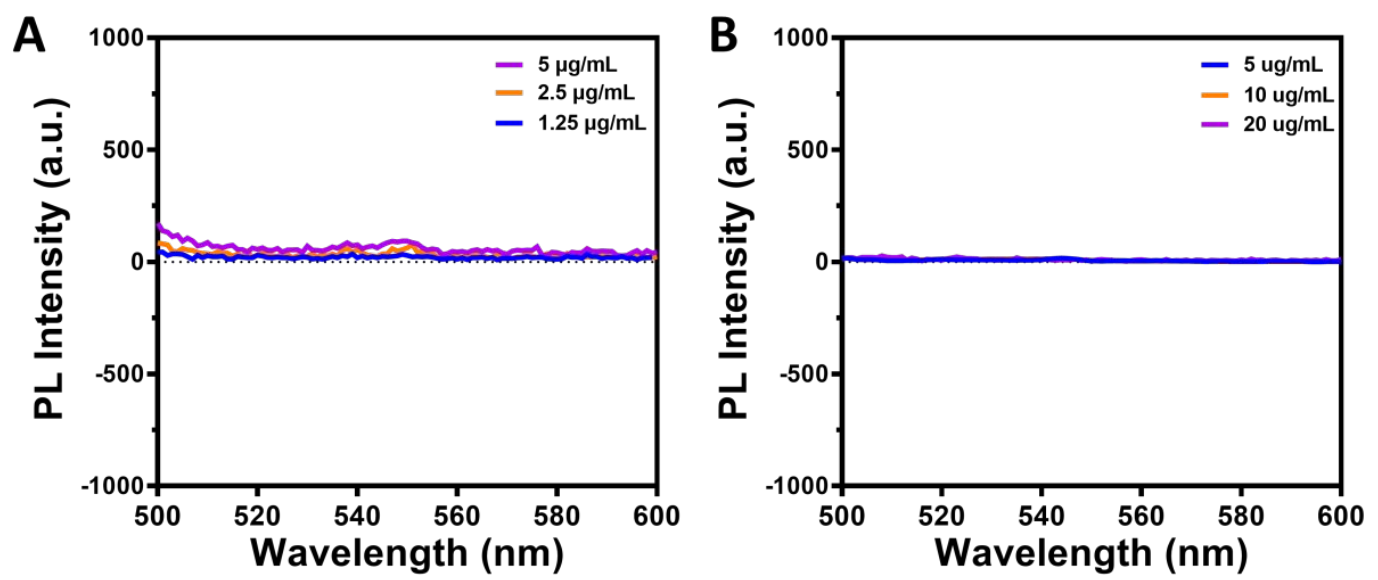

Figure S10. Evaluation the ROS generation by DCFH probe. (A) The fluorescence spectra of DCFH solutions with different concentrations of IR780@Pt NPs in the absence of laser irradiation. (B) The fluorescence spectra of DCFH solutions with different concentrations of Pt-CD (based on the content of CDDP).

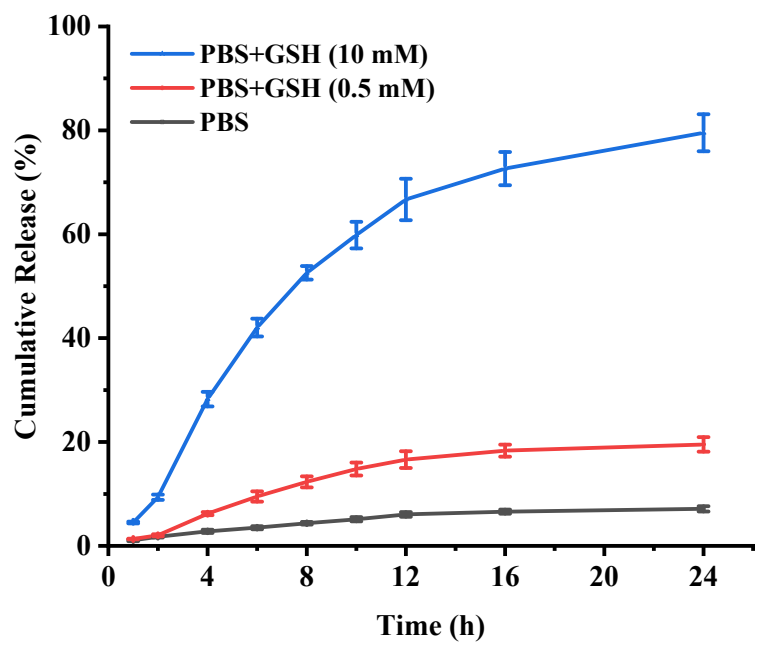

Figure S11. Cumulative release profile of IR780 from IR780@Pt NPs in solutions with different concentrations of GSH. 

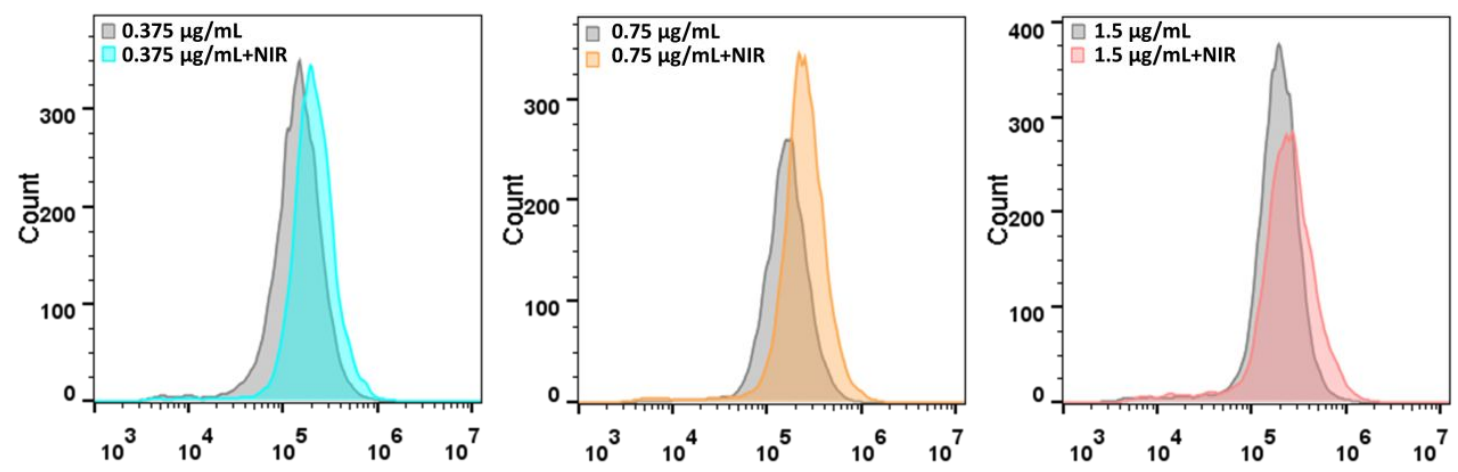

Figure S12. Flow cytometric quantification of the cellular ROS levels by ROS probe DCFH-DA.

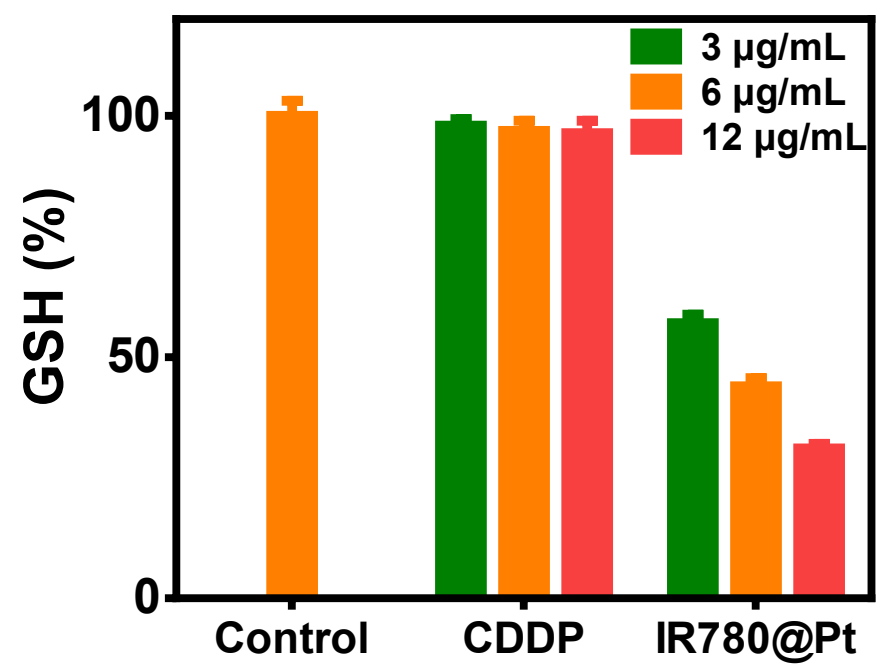

Figure S13. The intracellular GSH levels determined after incubation with CDDP or IR780@Pt NPs at various concentrations based on CDDP content. 


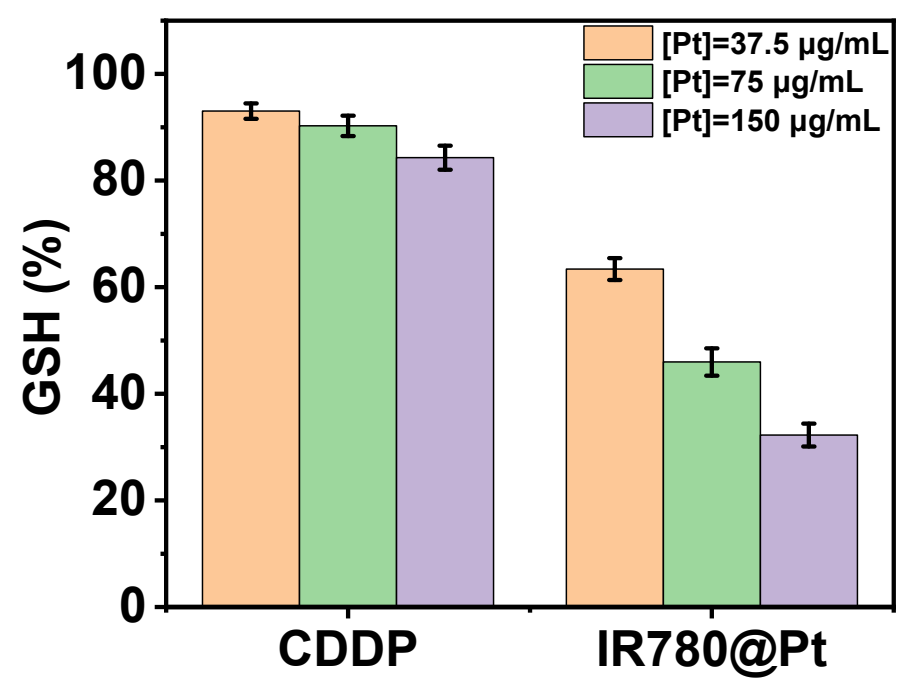

Figure S14. The GSH consumption property of CDDP and IR780@Pt NPs measured by GSH assay kit.
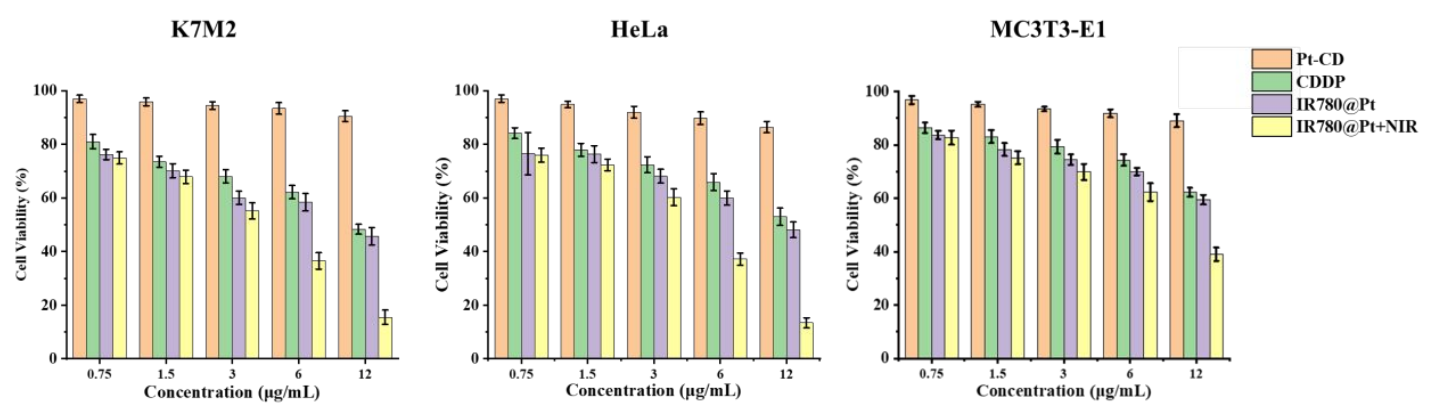

Figure S15. Cell viability of K7M2, HeLa and MC3T3-E1 cells determined by MTT assay after treated with various drugs with/without NIR irradiation. 


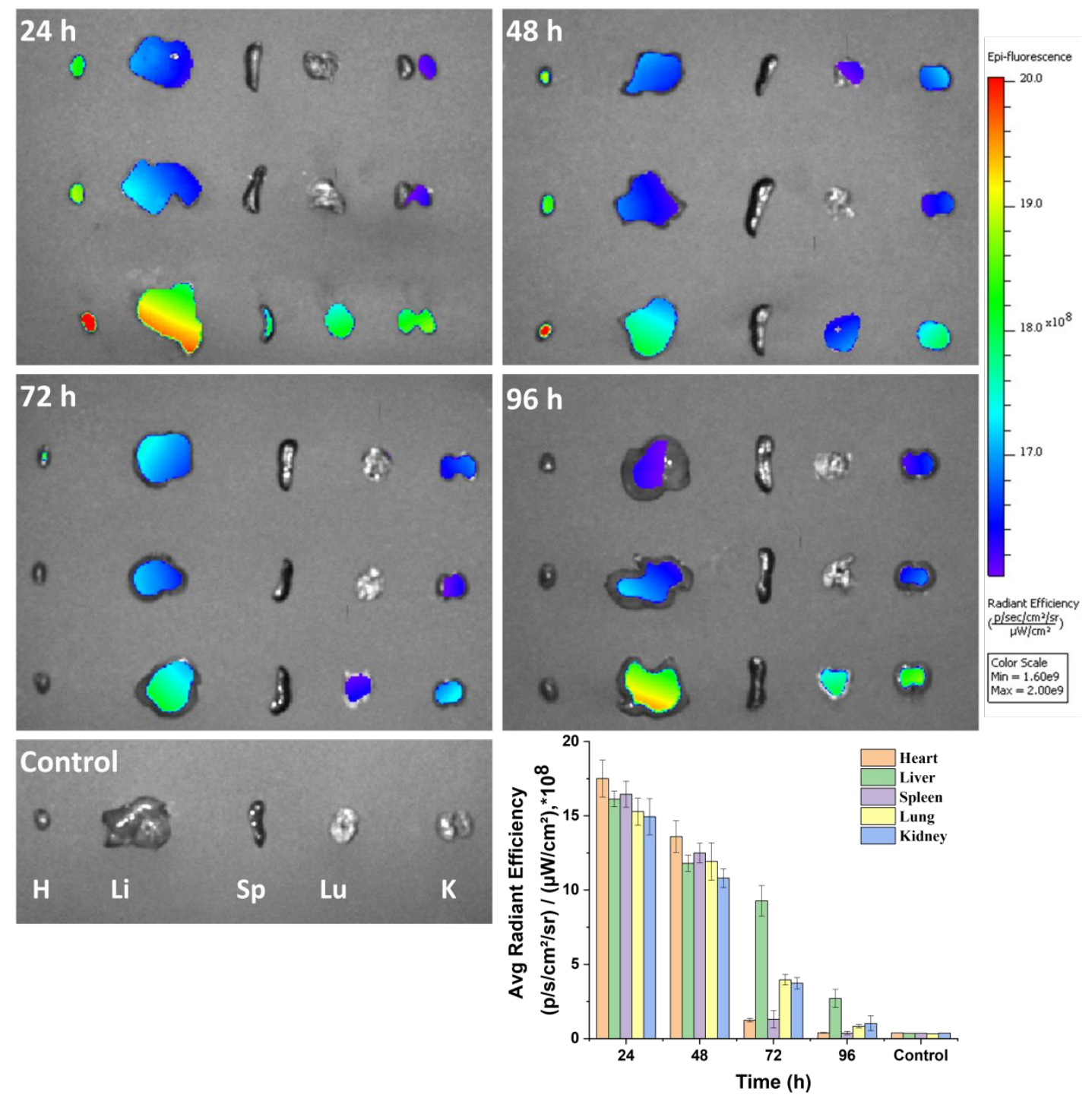

Figure S16. Evaluation of the in vivo metabolism of IR780@Pt NPs by tracking the fluorescence intensity in the main organs of mice after i.v. injection (dosage: $5 \mathrm{mg} / \mathrm{kg}$ ). H, heart; Li, liver, Sp, spleen; Lu, lung, K, kidney; T, tumor. 